\title{
Réflexion sur la pertinence de s'intéresser aux stratégies d'étude des étudiants ayant un trouble d'apprentissage ou un TDA/H afin de favoriser leur réussite scolaire
}

Auteur(s)

Jonathan Turcotte, UQAC, jonathan.turcotte1@uqac.ca

Manon Doucet, UQAC, Manon Doucet@uqac.ca

Marie-Pierre_Baron, UQAC, Marie-Pierre Baron@uqac.ca 


\section{$\&$}

\section{REVUE HYBRIDE DE L'ÉDUCATION}

\section{Résumé}

Depuis les deux dernières décennies, le nombre d'étudiants collégiaux ayant un trouble d'apprentissage (TA) ou un trouble du déficit de l'attention avec ou sans hyperactivité (TDA/H) a connu une augmentation fulgurante et il apparait de plus en plus urgent de se préoccuper davantage de leur réussite scolaire. L'un des principaux obstacles à la réussite identifié par ces étudiants est "le manque de technique pour étudier " (Wolforth et Roberts, 2010, p. 28). Cet article cherche à démontrer la nécessité de s'intéresser de près aux stratégies d'études employées par les étudiants collégiaux qui ont un TA ou un TDA/H afin de s'assurer que ces derniers utilisent des stratégies d'études efficaces.

Mots-clés: Stratégies d'étude ; réussite collégiale ; autorégulation des apprentissages ; TDA/H ; trouble d'apprentissage. 


\section{$\infty_{0}^{\circ}$ \\ REVUE HYBRIDE DE L'ÉDUCATION}

\section{Introduction}

L'année 2017 marque le cinquantième anniversaire des collèges d'enseignement général et professionnel (cégeps). C'est effectivement en 1967, à la suite des recommandations du rapport Parent, que les cégeps ont vu le jour afin de favoriser l'accès aux études postsecondaires. Le rôle des cégeps dans la démocratisation de l'éducation au Québec est non négligeable. En effet, le nombre d'étudiants québécois poursuivant des études collégiales passera de 18541 à 70385 entre 1967 et 1970, soit une augmentation de $380 \%$ en trois ans (Linteau, Durocher, Robert et Ricard, 1986). Cela fut possible, d'une part, en raison de son implantation rapide partout au Québec qui a permis la régionalisation des services éducatifs (Lessard et Brochu, 2013) et, d'autre part, parce que ce nouvel ordre d'enseignement unique en Amérique du Nord se distinguait clairement de l'école secondaire et de l'université en proposant deux types d'enseignement : la formation préparatoire aux études universitaires et la formation technique menant au marché du travail (Lessard et Brochu, 2013).

Depuis les années 1990, les cégeps font face à de nouveaux défis tels que la modification des cours obligatoires, la baisse démographique en région, l'introduction de nouvelles technologies, des coupes budgétaires importantes, l'alignement de la formation professionnelle au secondaire et de la formation technique au collégial, l'harmonisation de la formation collégiale et universitaire, l'évaluation institutionnelle, la remise en question de la formation générale au profit d'une formation adaptée à l'emploi, une forte préoccupation pour la réussite des étudiants et la diversification des étudiants s'inscrivant au cégep (Lessard et Brochu, 2013). Parmi ces enjeux, assurer la réussite d'un nombre toujours croissant d'étudiants en situation de handicap émergents (ÉSHÉ) constitue un défi important à relever pour la pérennité des établissements collégiaux (Vagneux et Girard, 2014). Avec l'avènement de nouvelles populations étudiantes, les étudiants en situation de handicap (ÉSH) se divisent désormais en deux catégories : la population traditionnelle et la population émergente. Les ÉSHÉ sont des étudiants ayant des troubles d'apprentissage, des troubles du déficit de l'attention avec ou sans hyperactivité, des troubles de santé mentale et des troubles du spectre de l'autisme. Inversement, les étudiants en situation de handicap traditionnels regroupent tous les autres ÉSH, notamment les étudiants ayant un handicap physique. Cette distinction s'avère pertinente dans l'optique où la population émergente connait une importante augmentation depuis les deux dernière décennies (Vagneux et Girard, 2014). Plus particulièrement, l'accroissement des étudiants ayant un trouble d'apprentissage (TA) ou un trouble du déficit de l'attention avec ou sans hyperactivité (TDA/H) force la communauté collégiale à réfléchir aux dispositifs à mettre en place pour soutenir la réussite de ces étudiants.

Cet article propose une réflexion sur la nécessité de s'intéresser à l'autorégulation des apprentissages en contexte d'étude. II est question de 


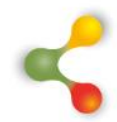

\section{REVUE HYBRIDE DE L'ÉDUCATION}

démontrer toute la pertinence que revêt l'utilisation de stratégies d'étude efficaces afin de favoriser la réussite scolaire de ces étudiants. Pour ce faire, un aperçu de l'historique de l'intégration et de l'inclusion des ÉSH ainsi que de l'évolution de la conception du handicap permet, d'abord, de dresser un portrait juste de la situation de cette catégorie d'étudiants au sein des établissements collégiaux. Ensuite, la question de la réussite scolaire des étudiants ayant un TA ou un TDA/H au niveau collégial est abordée. Enfin, en prenant en considération l'accentuation de l'autonomie des étudiants au niveau collégial, il est argumenté que l'utilisation de bonnes stratégies d'étude influence la réussite des étudiants ayant un TA ou un $\mathrm{TDA} / \mathrm{H}$.

\section{Mise en contexte}

$\mathrm{Si}$, dès sa création en 1967, le réseau collégial a accueilli quelques étudiants en situation de handicap, il est à noter qu'aucun service spécifique n'était offert pour favoriser leur intégration (Fédération étudiante collégiale du Québec [FECQ], 2010). C'est environ une décennie plus tard, en 1978, que la Loi assurant l'exercice des droits des personnes handicapées était adoptée par l'Assemblée nationale, ce qui a mené à la création de l'Office des personnes handicapées du Québec (OPHQ) dont l'objectif est de défendre et de promouvoir les intérêts des personnes handicapées, notamment en favorisant leur intégration scolaire, professionnelle et sociale (Ministère de l'Enseignement Supérieur et de la Science [MESS], 1992).

Ce n'est qu'au début des années 1980 que sera initiée la mise en place de réels efforts pour offrir des services éducatifs aux ÉSH dans ces établissements. En 1982, le cégep du Vieux Montréal et le cégep de SainteFoy sont désignés par la direction générale de l'enseignement collégial " pour accueillir les étudiants handicapés, pour implanter un ensemble de mesures leur permettant de compenser leurs limitations fonctionnelles et pour développer une expertise en la matière " (MESS, 1992, p. 6). L'expérience et l'offre de services de ces deux cégeps s'élargiront au fil des ans, notamment par l'établissement d'un réseau de répondants locaux présents dans chaque cégep, ce qui permettra le déploiement de services éducatifs adaptés pour l'ensemble des collèges publics québécois ( $F E C Q$, 2010).

II faudra toutefois attendre jusqu'en 1992 pour voir apparaitre le document Accueil et intégration des personnes handicapées au collégial publié par le MESS qui met en exergue la volonté d'intégrer les ÉSH, notamment par la proposition de services palliatifs. Dans son orientation visant l'accessibilité universelle des programmes d'études, le MESS (1992) écrit ceci :

Cette orientation signifie que l'admission à un programme d'études 


\section{6}

\section{REVUE HYBRIDE DE L'ÉDUCATION}

ou à un cours ne peut être refusée à une personne sur la base de sa déficience. Dans la mesure du possible, des services palliatifs doivent donc être mis en place pour permettre l'accès aux cours ou au programme choisi par l'élève qui satisfait aux exigences d'admission. Le refus d'admission d'une personne handicapée n'est justifiable que sur une base individuelle et que si le cégep peut démontrer, hors de tout doute, son impossibilité d'effectuer les aménagements qui permettraient à l'élève de compenser ses limitations fonctionnelles (p. 8).

Depuis le début des années 2000, la diversification des besoins des ÉSH s'est accompagnée d'un élargissement des services adaptés offerts dans les cégeps. D'ailleurs, alors que le nombre d'étudiants en situation de handicap traditionnel a augmenté de $275 \%$ en passant de 726 à 1994 étudiants entre 2007 et 2012, la population émergente s'est, quant à elle, décuplée, passant de 577 à 5593 étudiants pour la même période (Comité Interordres de Montréal, 2013). Plus spécifiquement encore, il semble que les étudiants ayant un TA ou un TDA/H inscrits au collégial constituent la catégorie pour laquelle l'augmentation fut la plus importante depuis le milieu des années 2000 (Bonnelli, Ferland-Raymond et Campeau, 2010, p. 23). Cet essor d'une population estudiantine émergente, notamment les étudiants ayant un TA ou un TDA/H, a entrainé une reconfiguration des services adaptés qui étaient, jusque-là, focalisés sur les accommodements aux handicaps visibles. Cette nouvelle population étudiante a transformé le visage des services adaptés des cégeps (Beaumont et Lavallée, 2012). Effectivement, alors que le document Accueil et intégration des personnes handicapées au collégial (MESS, 1992) préconisait « davantage [une] accessibilité physique et [une] accessibilité à l'information en médias substituts [en précisant] qu'il appartiendra à la personne handicapée [...] de prendre les mesures nécessaires pour se doter d'une méthode efficace d'apprentissage » (p. 9), le contexte actuel semble plutôt mettre en lumière que les besoins particuliers de la population émergente nécessitent également le recours à des adaptations pédagogiques (Beaumont et Lavallée, 2012).

\section{Problématique}

$\underline{\text { Une transition entre le secondaire et le collégial difficile }}$

II appert que les différences importantes entre l'école secondaire et le cégep compliquent l'entrée aux études de plusieurs nouveaux étudiants, notamment les ÉSH. Filion (1990) souligne d'ailleurs que «l'un des facteurs explicatifs de l'échec et de l'abandon des études collégiales retenu par le Conseil des collèges dans son rapport de la fin des années 80 est ce qu'il appelait "le choc du passage du secondaire au collégial" " (p. 53). Et pour cause, cette transition entre l'école secondaire et le cégep se caractérise traditionnellement par un accroissement de l'autonomie des étudiants et une augmentation des responsabilités liées à une charge de 


\section{6}

\section{REVUE HYBRIDE DE L'ÉDUCATION}

travail accrue en dehors de la classe (Brochu et Moffet, 2010 ; Chouinard, Goulet et Tremblay, 2013 ; Métayer, 1991). Aussi, il importe de préciser que, n'étant plus soumis à l'obligation légale de fréquenter l'école, les étudiants de niveau collégial poursuivent volontairement leurs études, et ce, dans un contexte où les parents sont de moins en moins impliqués dans la scolarisation de leur enfant. Pour les étudiants en situation de handicap, cela signifie non seulement qu'ils sont libres de refuser l'ensemble des services d'aide des collèges, mais que ce sont généralement eux qui devront entreprendre les démarches pour obtenir des services (Chouinard et al., 2013). Ce désencadrement du processus d'apprentissage qu'amène la transition secondaire-collégial constitue un motif pour les étudiants d'accroitre leur autonomie par rapport à leurs apprentissages. Cependant, encore faut-il qu'ils soient en mesure de mobiliser les ressources cognitives nécessaires à la gestion autonome de leurs apprentissages.

Concernant plus spécifiquement les cégépiens présentant un TA ou un TDA/H, l'entrée au collège entraine des difficultés supplémentaires concernant la capacité de concentration, la sélection des informations importantes en classe, l'utilisation de stratégies pour réussir un examen et la gestion du temps (Connor, 2012). D'ailleurs, dans une étude menée par DuPaul, Pinho, Pollack, Gormley, et Laracy (2017) aux États-Unis, les étudiants ayant un TA ou un TDA/H qui sont en période de transition entre le secondaire et l'université rapportaient un plus grand niveau de désengagement scolaire et des relations interpersonnelles plus difficiles en comparaison à leurs comparses.

Quoi qu'il en soit, il semble que l'accroissement de l'autonomie amené par l'entrée au cégep, la charge de travail accrue, ainsi que la complexification des apprentissages à réaliser rendent la transition entre le secondaire et le collégial difficile, et ce, de façon encore plus importante chez les étudiants ayant un TDA/H ou un TA. Dans cette optique, il apparait essentiel de se pencher sur l'utilisation de stratégies favorisant l'apprentissage employées de façon autonome par les étudiants ayant un TA ou un TDA/H.

\section{$\underline{\text { Réussite scolaire des étudiants ayant un TA ou un TDA/H }}$}

Généralement, pour diagnostiquer un TA ou un TDA/H, les différentes manifestations de ces troubles doivent être présentes dans plus d'un contexte (American Psychiatric Association, 2013, 2015). II va de soi que l'école constitue souvent l'un de ces contextes et il est donc logique que les élèves et les étudiants qui ont un TA ou un TDA/H connaissent généralement des difficultés importantes sur le plan scolaire. Si, par sa définition même, le TA implique des difficultés scolaires importantes, il appert que les problèmes liés à l'attention et à la mémoire de travail qui sont associés au TDA/H (Rao, Alderson, Kasper, Hudec et Patros, 2013) entraine aussi des difficultés en lecture, en écriture et en mathématique (Bonnelli et al., 2010). 


\section{$\&$}

\section{REVUE HYBRIDE DE L'ÉDUCATION}

En ce qui a trait plus spécifiquement aux cégépiens, Jorgensen, Fichten, Havel, Lamb, James et Barile (2005) ont réalisé une étude sur la réussite scolaire des étudiants collégiaux québécois en situation de handicap sur une période de douze ans. Les résultats de l'étude montrent qu'il n'y a pas de différence significative entre les taux de diplomation et les résultats scolaires des ÉSH et des autres étudiants, alors que plus de la moitié de ces ÉSH faisaient partie de la catégorie "Learning disability/ADD». Toutefois, lorsque l'on compare les étudiants ayant un TA ou un TDA/H aux autres ÉSH, on remarque qu'ils sont moins performants. On constate toutefois une différence non significative des résultats des étudiants sans handicap $(80,5 \%)$ en comparaison à ceux des étudiants ayant un TA ou un TDA/H (78,3\%). Aussi, les auteurs notent que les ÉSH se dotent généralement d'un horaire moins chargé et prennent une session de plus que leurs comparses pour obtenir un diplôme.

Bien que ces résultats soient encourageants quant à la poursuite des études supérieures pour les étudiants ayant un TA ou un TDA/H, l'étude a été publiée il y a plus de 10 ans et, depuis cette époque, le nombre d'étudiants ayant un TA ou un TDA/H a augmenté de façon draconienne. D'ailleurs, il faut spécifier que ces résultats concernent uniquement les étudiants ayant un TA ou un TDA/H qui ont réussi à accéder aux études postsecondaires et que, de ce fait, tous ceux qui n'ont pas poursuivi leurs études ne sont pas considérés par l'étude. Or, l'hyperactivité et l'inattention sont considérées comme des facteurs de risque importants du décrochage scolaire (Gregg, 2009; Kent et al., 2011; Pica, Plante et Traoré, 2014 ; Vitaro, Brendgen, Larose et Tremblay, 2005). Les travaux de Barkley, Murphy et Fischer (2008) indiquent que les personnes ayant un TDA/H ont moins de chance de poursuivre des études postsecondaires que leurs pairs. Dans le même ordre d'idées, les élèves ayant un trouble d'apprentissage sont également plus à risque de décrocher de l'école secondaire (Fortin et Picard, 1999 ; Dunn, Chambers et Rabren, 2004 ; Gregg, 2009) et sont moins susceptibles d'avoir terminé des études postsecondaires que le reste de la population (Cortiella et Horowitz, 2014 ; Newman et al., 2011). Il est donc probable que la raison pour laquelle les étudiants ayant un TA ou un TDA/H réussissent aussi bien dans l'étude de Jorgensen et ses collaborateurs (2005) est que seuls les plus performants d'entre eux se rendent au niveau collégial.

Par ailleurs, aux États-Unis, on constate que les étudiants ayant un TDA/H sont plus susceptibles de s'inscrire à un parcours postsecondaire plus court (Kuriyan et al., 2013), d'obtenir un échec, d'abandonner un cours et de ne pas compléter leur programme d'étude en comparaison aux autres étudiants (Weyandt et al., 2013). Les étudiants ayant un TA vivent sensiblement les mêmes difficultés. Ils s'inscrivent à des programmes d'étude plus courts, leur moyenne cumulative est généralement plus faible (2,2 contre 2,7 pour l'ensemble des étudiants) et ils échouent davantage de cours (Cortiella et Horowitz, 2014). Bref, il semble donc que les étudiants 


\section{6}

\section{REVUE HYBRIDE DE L'ÉDUCATION}

ayant un TA ou un TDA/H ont davantage de difficultés sur le plan scolaire, ce qui justifie que l'on s'emploie à mettre en place des services visant à favoriser leur réussite. Les établissements d'enseignement postsecondaire québécois offrent donc de plus en plus de services destinés aux étudiantes et aux étudiants ayant un TA ou un TDAH, sans toutefois que l'efficacité des mesures mises en place soit étayée par des données probantes (Wolforth et Roberts, 2010).

Parmi les services proposés à ces étudiants, on retrouve, le plus souvent, l'ajout de temps supplémentaires pour les examens et les travaux, la réduction du nombre de cours et l'allongement de la durée du programme, les mesures d'accommodement liées au cours (p. ex. prise de notes par un pair, livre audio), l'utilisation de technologies d'assistances et les aides technologiques (Wolforth et Roberts, 2010). Or, en s'inspirant d'une vision sociale du handicap, le processus de production du handicap (Fougeyrollas, 1995, 1998, 2010) vise « le renforcement des aptitudes et la compensation des incapacités par la réadaptation, mais également la réduction des obstacles présents dans les milieux de vie " (Centre de documentation collégiale, 2013, p. 3). Il s'agit donc évidemment de modifier les caractéristiques environnementales ${ }^{1}$ de l'apprenant, mais tout en continuant à agir sur ses caractéristiques personnelles ${ }^{2}$ afin de le placer dans une situation qui permet le développement et l'expression de son plein potentiel. II semble néanmoins que les services proposés dans les établissements d'enseignement postsecondaire afin d'aider les étudiants présentant un TA ou TDA/H soient focalisés sur l'attribution de ressources externes ou environnementales au détriment de l'amélioration des stratégies et des ressources internes de l'apprenant. D'ailleurs, dans la recherche sur la situation des étudiants présentant un TA ou un TDA/H qui fréquentent les cégeps au Québec de Wolforth et Roberts (2010), l'un des principaux obstacles identifiés par ces étudiants est «le manque de technique pour étudier » (p. 28). II convient donc de s'intéresser de près aux stratégies d'étude qui sont actuellement mobilisées par les étudiants ayant un TA ou un TDA/H.

\section{$\underline{\text { Stratégies d'étude et réussite scolaire au collégial }}$}

L'accroissement de l'autonomie que provoque l'entrée au cégep et la préoccupation de la réussite de tous les étudiants amènent à réfléchir

\footnotetext{
1 Ces modifications des caractéristiques environnementales sont les mesures d'accommodation (p. ex. l'octroi de temps supplémentaire, l'utilisation d'aide technologique) dédiées aux étudiants ayant un TA ou un TDA/H dans le but de favoriser leur réussite.

2 Ici, par caractéristiques personnelles de l'étudiant, on fait référence à ces ressources cognitives, métacognitives et affectives qui permettent de mieux gérer ses apprentissages (p. ex. mobilisation de ses stratégies d'étude efficaces, utilisation de stratégies de gestion du temps, gestion de son sentiment de compétence, etc.).
} 


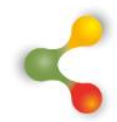

\section{REVUE HYBRIDE DE L'ÉDUCATION}

aux divers moyens que les étudiants ayant un TA ou un TDA/H peuvent utiliser pour mieux gérer leurs apprentissages de façon autonome. Si le cégep exige de ces étudiants qu'ils consacrent une bonne partie de leur temps personnel à l'apprentissage, on dispose actuellement de peu de données sur la façon dont ils s'y prennent (Gagnon, 2014). Or, l'un des éléments essentiels de la régulation autonome des apprentissages est le choix de stratégies d'étude (Nelson et Narens, 1990). De façon générale, la planification stratégique de l'étude apparait être essentielle à la gestion des apprentissages (Eshel et Kohavi, 2003; Griffin, MacKewn, Moser et VanVuren, 2012 ; Schraw et Dennison, 1994 ; Weinstein et Palmer, 2002 ; Wrugt et Oort, 2008 ; Young et Fry, 2008). Les compétences liées à l'étude sont d'ailleurs fréquemment corrélées à des résultats scolaires plus élevés (Elliot, McGregor et Gable, 1999 ; Knouse, Rawson, Vaughn \& Dunlosky, 2015 ; Nonis \& Hudson, 2010 ; Peverly, Brobst, Graham, \& Shaw, 2003; Robbins, Lauver, Le, Davis, Langley et Carlstrom, 2004 ; West et Sadoski, 2011 ; Yip, 2007). Une étude de Griffin et coll. (2012) suggère même que la différence de performance scolaire entre les garçons et les filles (les filles performent généralement mieux que les garçons) pourrait s'expliquer par des compétences d'études significativement plus élevées pour les filles. Après avoir contrôlé les variables de compétence pour l'étude, les auteurs ont montré que la différence de performance ne pouvait plus être seulement expliquée par le genre des étudiants. Quoi qu'il en soit, il semble y avoir un besoin d'étudier davantage ces variables relatives à l'apprentissage autonome, notamment en ce qui concerne l'utilisation de stratégies d'étude chez les étudiants collégiaux (Murray et Wren, 2003).

Concernant les étudiants présentant un TA ou un TDA/H, Connor (2012) affirme que l'enseignement explicite de stratégies d'étude aux élèves du primaire et du secondaire qui ont un TA ou un TDA/H constitue une fondation solide pour réussir la transition vers le niveau collégial. Shaw, Madaus et Banerjee (2009) mentionnent même que les stratégies d'apprentissage relatives à l'étude devraient être enseignées de façon à ce que les étudiants soient en mesure de les utiliser de façon autonome. Pourtant, malgré cette nécessité d'acquérir, de maintenir et de généraliser des stratégies d'étude efficace, il semble que les adolescents ayant un TA n'ont pas appris à étudier lors de leur parcours scolaire obligatoire (Alley et Deshler, 1979 ; Reid, 1988, cités dans Benz, Fabian et Nelson, 1996). Dans le même sens, il semble que les étudiants collégiaux qui ont un TDA/H éprouvent de nombreuses difficultés en ce qui concerne l'utilisation adéquate de stratégies d'étude (Reaser, Prevatt, Petscher et Proctor, 2007 ; Weyandt et al., 2013). Advokat, Lane et Luo (2011) notent qu'en général :

Les étudiants ayant un TDA/H ont une moyenne cumulative plus basse, se retirent de plus de cours et ne prennent pas autant de notes que les autres étudiants. Ils n'étudient pas plus longtemps, ou plus intensément, et la plupart n'étudient pas leur examen à l'avance (traduction libre, p. 664). 


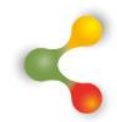

\section{REVUE HYBRIDE DE L'ÉDUCATION}

Ces auteurs suggèrent même "que si les étudiants ayant un TDA/H parvenaient à développer les compétences organisationnelles nécessaires et la discipline inhérente à l'utilisation de bonnes habitudes d'étude, ils pourraient surmonter la différence de réussite scolaire sans utiliser de médicaments stimulants » (traduction libre, p. 664).

Dans l'ensemble, il appert que la façon dont s'y prennent les étudiants ayant un TA ou un TDA/H pour étudier influence leur réussite scolaire. Cela n'est pas très surprenant considérant que l'étude autonome occupe une place considérable dans la poursuite des études au niveau postsecondaire. Le peu de données disponibles sur le sujet semble indiquer que ces étudiants n'ont pas appris à gérer efficacement leur étude, et ce, malgré une pertinence évidente. Dans cette optique, il s'avère opportun de s'intéresser plus spécifiquement aux stratégies d'étude que ces étudiants utilisent afin de favoriser leur réussite. II faut toutefois noter qu'il existe des différences importantes concernant le rendement des différentes stratégies d'étude menant à l'apprentissage (Dunlosky, Rawson, Marsh, Nathan et Willingham, 2013 ; Gagnon, 2014).

En effet, les ressources mises à la disposition des collégiens présentent souvent une série de stratégies, sans offrir d'information spécifique quant à leur performance relative et leurs limites respectives. Or, ceci peut amener l'étudiant à croire que toute technique a son utilité et qu'il s'agit d'une question de préférence ou de style personnel. Toutefois, une telle conclusion serait inexacte. En effet, les études en sciences de l'apprentissage suggèrent qu'il n'existe qu'une minorité de techniques dont le rendement est maximal (Gagnon, 2014, p. 11).

Bref, il semble que parmi un large éventail de stratégies d'étude proposées et utilisées, seules quelques-unes d'entre elles sont considérées comme efficaces par les recherches en psychologie cognitive et en éducation.

\section{Apports et prospectives}

Devant le peu d'information quant aux stratégies d'études utilisées par les étudiants ayant un TA ou un TDA/H, devant la hausse de la population d'étudiants ayant un TA ou un TDA/H et puisqu'il est reconnu qu'il existe des stratégies d'étude qui sont plus efficaces que d'autres (Dunlosky et al., 2013), il apparait essentiel de s'intéresser de près aux stratégies d'étude employées par les étudiants collégiaux qui ont un TA ou un TDA/H afin de s'assurer que ces derniers utilisent des stratégies d'étude efficaces. C'est pourquoi, dans le cadre du projet de mémoire de la maitrise en éducation, sera réalisé une recherche visant à identifier les stratégies d'étude déclarées par ces étudiants dans le but de les comparer aux stratégies d'étude identifiées comme efficaces. La recherche permettra également de vérifier la présence d'un lien entre l'utilisation de stratégies d'étude efficaces et la réussite scolaire des étudiants ayant un TA ou un 


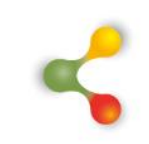

\section{REVUE HYBRIDE DE L'ÉDUCATION}

TDA/H. L'approfondissement des connaissances concernant les stratégies d'étude des étudiants de niveau collégial qui ont un TA ou un TDA/H est une avenue prometteuse pour favoriser la réussite de ces jeunes. Ultimement, les résultats de cette recherche pourront servir à réaliser des activités d'enseignement pour promouvoir l'utilisation de stratégies d'étude efficaces chez ces étudiants. 


\section{REVUE HYBRIDE DE L'ÉDUCATION}

\section{Références}

Advokat, C., Lane, S. M., et Luo, C. (2011). College Students With and Without ADHD. Journal of Attention Disorders, 15(8), 656-666. doi:10.1177/1087054710371168

American Psychiatric Association. (2013). Diagnostic and statistical manual of mental disorders : DSM-5 (5th ed.). Arlington, Virg.: American Psychiatric Association.

American Psychiatric Association. (2015). DSM-5 : manuel diagnostique et statistique des troubles mentaux ( $5^{\mathrm{e}}$ ed.). Issy-les-Moulineaux : Elsevier Masson.

Barkley, R. A., Murphy, K. R., et Fischer, M. (2008). ADHD in adults: What the science says. New York, NY: Guilford Press.

Beaumont, J., et Lavallée, C. (2012). L'enseignant-ressource: "Un prof qui parle aux profs". Pédagogie collégiale, 25(4), 27-31

Benz, C. R., Fabian, M. P., et Nelson, W. R. (1996). Assessing Study Skills of Students with Learning Disabilities. Clearing House, 69(6), 349353.

Bonnelli, H., Ferland-Raymond, A. E., et Campeau, S. (2010). Portrait des étudiants et étudiantes en situation de handicap et des besoins émergents à renseignement postsecondaire. Une synthèse des recherches et de la consultation : version abrégée. Québec : Ministère de l'Éducation, du Loisir et du Sport.

Brochu, É., et Moffet, J. D. (2010). Regards renouvelés sur la transition entre le secondaire et le collégial. Québec : Conseil supérieur de l'éducation.

Centre de documentation collégiale. (2013). Étudiants en situation de handicap, diversité et inclusion. Bulletin de la documentation collégiale, 11. Repéré à http://www.cdc.qc.ca/bulletin/bulletin-CDC11-etudiants-situation-handicap-nov-2013.pdf

Connor, D. J. (2012). Helping students with disabilities transition to college. Teaching Exceptional Children, 44(5), 16-25.

Chouinard, J., Goulet, L. et Tremblay, M. (2013). Les aides technologiques et la quête d'autonomie des élèves ayant des besoins particuliers : un apport déterminant. Association québécoise des troubles d'apprentissage. Repéré à http://institutta.com/les-aides- 


\section{8}

\section{REVUE HYBRIDE DE L'ÉDUCATION}

technologiques-et-la-quete-dautonomie-des-eleves-ayant-desbesoins-particuliers-un-apport-determinant/

Comité Interordres de Montréal (2013). Intégrer les nouvelles populations étudiantes en situation de handicap; mission possible! Repéré à https://docs.google.com/file/d/0B2z2oz4ckHW6RldJYW5YcVEwNjQ ledit

Cortiella, C., et Horowitz, S. H. (2014). The state of learning disabilities: Facts, trends, and emerging issues. New York, NY: National Center for Learning Disabilities.

Ducharme, D., et Montminy, K. (2012). L'accommodement des étudiants et étudiantes en situation de handicap dans les établissements d'enseignement collégial. Québec : Commission des droits de la personne et des droits de la jeunesse (CDPDJ).

Dunn, C., Chambers, D., \& Rabren, K. (2004). Variables Affecting Students' Decisions to Drop Out of School. Remedial and Special Education, 25(5), 314-323.

Dunlosky, J., Rawson, K. A., Marsh, E. J., Nathan, M. J., \& Willingham, D. T. (2013). Improving Students' Learning With Effective Learning Techniques. Psychological Science in the Public Interest, 14(1), 458. doi:10.1177/1529100612453266

DuPaul, G. J., Gormley, M. J., \& Laracy, S. D. (2013). Comorbidity of LD and ADHD. Journal of Learning Disabilities, 46(1), 43-51. doi:10.1177/0022219412464351

Elliot, A., McGregor, H., \& Gable, S. (1999). Achievement goals, study strategies, and exam performance: A mediational analysis. Journal of Educational Psychology, 91(3), 549-563.

Eshel, Y., \& Kohavi, R. (2003). Perceived Classroom Control, SelfRegulated Learning Strategies, and Academic Achievement. Educational Psychology, 249-260. doi:10.1080/0144341032000060093

Fédération étudiante collégiale du Québec. (2010). Pour une éthique de l'égalité des chances : recherche sur les étudiants avec besoins particuliers. 62e congrès ordinaire, Collège Ahunstic.

Filion, A. (1990). La réussite et la diplomation au collégial : Des chiffres et des engagements. Montréal : Fédération des cégeps.

Fortin, L. \& Picard, Y. (1999). Les élèves à risque de décrochage scolaire : facteurs discriminants entre décrocheurs et persévérants. Revue des sciences de l'éducation, 25(2), 359-374. doi:10.7202/032005ar 


\section{REVUE HYBRIDE DE L'ÉDUCATION}

Fougeyrollas, P. (1995). Le processus de production culturelle du handicap : contextes sociohistoriques du développement des connaissances corporelles et fonctionnelles. Lac St-Charles, (Québec) : CqcidihSccidih.

Fougeyrollas, P. (1998). Classification québécoise : processus de production $d u$ handicap. Lac St-Charles, (Québec): Réseau international sur le processus de production du handicap.

Fougeyrollas, P. (2010). La funambule, le fil et la toile : transformations réciproques du sens du handicap. Québec: Presses de l'Université Laval.

Gagnon, M. (2014). La connaissance des stratégies d'apprentissage chez les collégiens. Montréal: Association des collèges privés du Québec. Repéré à https://www.acpq.net/static/uploaded/Files/documents/recherches/r apport-prep-mathieu-gagnon.pdf

Griffin, R., MacKewn, A., Moser, E., \& VanVuren, K. W. (2012). Do Learning and Study Skills Affect Academic Performance?--An Empirical Investigation. Contemporary Issues in Education Research, 5(2), 109-116.

Gregg, N. (2009). Adolescents and adults with learning disabilities and ADHD: assessment and accommodation. New York: The Guilford Press.

Jorgensen, S., Fichten, C. S., Havel, A., Lamb, D., James, C., \& Barile, M. (2005). Academic Performance of College Students with and without Disabilities: An Archival Study. Canadian Journal of Counselling, 39(2), 101-117.

Kent, K. M., Pelham, W. E., Molina, B. S. G., Sibley, M. H., Waschbusch, D. A., Yu, J., . . Karch, K. M. (2011). The Academic Experience of Male High School Students with ADHD. Journal of Abnormal Child Psychology, 39(3), 451-462. doi:10.1007/s10802-010-9472-4

Kuriyan, A. P., Pelham, W. E., Molina, B. S. G., Waschbusch, D. A., Gnagy, E. M., Sibley, M. H., ... Kent, K. M. (2013). Young adult educational and vocational outcomes of children diagnosed with ADHD. Journal of Abnormal Child Psychology, 41, 27-41.

Knouse, L. E., Rawson, K. A., Vaughn, K. E., et Dunlosky, J. (2015). Does Testing Improve Learning for College Students With AttentionDeficit/Hyperactivity Disorder? Clinical Psychological Science, 4(1), 136-143. doi:10.1177/2167702614565175 


\section{REVUE HYBRIDE DE L'ÉDUCATION}

Lessard, C. et Brochu, E. (2013). Collège d'enseignement général et professionnel (CEGEP). Toronto: Encyclopédie canadienne. Repéré à http://www.encyclopediecanadienne.ca/fr/article/collegedenseignement-general-et-professionnel-cegep/

Linteau, P.-A., Robert, J.-C., Durocher, R., \& Ricard, F. (1979). Histoire du Quebec contemporain. Montréal: Boréal Express.

Macé, A.L. et Rivard, M.P. (2013). Pratiques des services d'aide à l'apprentissage auprès des nouvelles populations en situation de handicap dans les établissements postsecondaires. Projet Interordres, Consortium d'animation sur la persévérance et la réussite en enseignement supérieur.

Ministère de l'Enseignement Supérieur et de la Science. (1992). Accueil et intégration des personnes handicapées au collégial. Québec: Gouvernement du Québec.

Métayer, M. (1991). La transition du secondaire au cégep. Montréal, Québec : Collège Lionel-Groulx.

Murray, C., et Wren, C. (2003). Cognitive, Academic, and Attitudinal Predictors of the Grade Point Averages of College Students with Learning Disabilities. Journal of Learning Disabilities, 36(5), 407415.

Nelson, T. O. (1990). Metamemory: A Theoretical Framework and New Findings. Dans G. H. Bower (Ed.), Psychology of Learning and Motivation (p. 125-173). New York: Academic Press.

Newman, L., Wagner, M., Knokey, A.-M., Marder, C., Nagle, K., Shaver, D., ... Wei, X. (2011). The post-high school outcomes of young adults with disabilities up to 8 years after high school. A report from the National Longitudinal Transition Study-2 (NLTS2) (NCSER 20113005). Menlo Park, CA: SRI International.

Nonis, S., \& Hudson, G. (2010). Performance of College Students: Impact of Study Time and Study Habits. Journal of Education for Business, 85(4), 229-238. doi:10.1080/08832320903449550

Office des personnes handicapées du Québec. (1984). À part... égale. L'intégration sociale des personnes handicapées : un défi pour tous. Québec : Les Publications du Québec.

Peverly, S. T., Brobst, K. E., Graham, M., \& Shaw, R. (2003). College Adults Are Not Good at Self-Regulation: A Study on the Relationship of Self- 


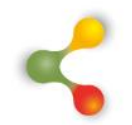

\section{REVUE HYBRIDE DE L'ÉDUCATION}

Regulation, Note Taking, and Test Taking. Journal of Educational Psychology, 95(2), 335-346. doi:10.1037/0022-0663.95.2.335

Pica, L. A., Plante, N. et Traoré, I. (2014). Décrochage scolaire chez les élèves du secondaire du Québec, santé physique et mentale et adaptation sociale: une analyse des principaux facteurs associés. Zoom santé, (46), 1-20.

Proctor, B. E., Prevatt, F. F., Adams, K., Reaser, A., \& Petscher, Y. (2006). Study Skills Profiles of Normal-Achieving and AcademicallyStruggling College Students. Journal of College Student Development, 47(1), 37-51.

Rao, S. M., Alderson, R. M., Kasper, L. J., Hudec, K. L., \& Patros, C. H. G. (2013). Attention-Deficit/Hyperactivity Disorder (ADHD) and Working Memory in Adults: A Meta-Analytic Review. Neuropsychology, 27(3), 287-302. doi:10.1037/a0032371

Reaser, A., Prevatt, F., Petscher, Y., \& Proctor, B. (2007). The learning and study strategies of college students with ADHD. Psychology in the Schools, 44(6), 627-638. doi:10.1002/pits.20252

Robbins, S. B., Lauver, K., Le, H., Davis, D., Langley, R., \& Carlstrom, A. (2004). Do Psychosocial and Study Skill Factors Predict College Outcomes? A Meta-Analysis. Psychological Bulletin, 130(2), 261288.

Schraw, G., Dennison, R. S. (1994). Assessing metacognitive awareness. Contemporary Educational Psychology, 19, 460-475.

Shaw, S. F., Madaus, J. W., et Banerjee, M. (2009). Enhance Access to Postsecondary Education for Students With Disabilities. Intervention in School and Clinic, 44(3), 185-190. doi:10.1177/1053451208326047

Vagneux, S. et Girard, S. (2014) Les étudiants en situation de handicap émergents à l'université : état de situation et pistes d'action. Québec, $Q C$ : Le réseau de l'Université du Québec.

Vitaro, F., Brendgen, M., Larose, S., \& Tremblay, R. E. (2005). Kindergarten Disruptive Behaviors, Protective Factors, and Educational Achievement by Early Adulthood. Journal of Educational Psychology, 97(4), 617-629. doi:10.1037/0022-0663.97.4.617

Weinstein, C. E., et Palmer, D. R. (2002). LASSI. User's Manual for those administering Learning and Study Strategies Inventory (2nd Edition). Clearwater, FL: H\&H Publishing Company. 


\section{$\&$}

\section{REVUE HYBRIDE DE L'ÉDUCATION}

West, C., \& Sadoski, M. (2011). Do study strategies predict academic performance in medical school? Medical Education, 45(7), 696-703. doi:10.1111/j.1365-2923.2011.03929.x

Weyandt, L. L., DuPaul, G. J., Verdi, G., Rossi, J. S., Swentosky, A., Vilardo, B. A., ... Carson, K. M. (2013). The performance of college students with and without ADHD: Neuropsychological, academic, and psychosocial functioning. Journal of Psychopathology and Behavioral Assessment, 35, 421-435.

Wolforth, J., et Roberts, E. (2010). La situation des étudiantes et des étudiants présentant un trouble d'apprentissage ou un trouble de déficit de l'attention avec ou sans hyperactivité qui fréquentent les cégeps au Québec : ce groupe a-t-il un besoin légitime de financement et de services? Québec : ministère de l'Éducation, du Loisir et du Sport.

Wrugt, A., et Oort, F. J. (2008). Metacognition, achievement goals, study strategies and academic achievement: pathways to achievement. Metacognition and Learning, 3(2), 123-146.

Yip, M. W. (2007). Differences in Learning and Study Strategies between High and Low Achieving University Students: A Hong Kong study. $\begin{array}{lll}\text { Educational } \quad \text { Psychology, 27(5), 597-606. } & \end{array}$ doi:10.1080/01443410701309126

Young, A., \& Fry, J. D. (2008). Metacognitive awareness and academic achievement in college students. Journal of the Scholarship of Teaching and Learning, 8(2), 1-10. 